ISSN 0103-8478

\title{
Desenvolvimento de espessante alimentar com valor nutricional agregado, destinado ao manejo da disfagia
}

\author{
Development of food thickener with aggregated nutritional value, \\ for the management of dysphagia
}

\section{Carlos Henrique Pagno ${ }^{\mathrm{I}}$ Lucéia Fatima Souza ${ }^{\mathrm{I}}$ Simone Hickmann Flores $^{\mathrm{*}}$ Erna Vogt de Jong ${ }^{\mathrm{I}}$}

RESUMO

A disfagia se caracteriza por uma disfunção no processo de deglutição, sinal prevalente de doenças degenerativas como esclerose múltipla e doença de Parkinson. Alimentos de textura modificada e bebidas espessadas são tradicionalmente utilizados no auxílio para o tratamento clínico da disfagia. Assim, este trabalho objetivou desenvolver uma formulação de espessante alimentar com valor nutricional agregado, e avaliar sua eficiência em diferentes alimentos líquidos (leite integral, sucos de maçã, uva e laranja), sob diferentes condições de tempo de preparo (10 e 120 minutos e 24 horas) e temperatura ambiente $\left(25^{\circ} \mathrm{C}\right)$ e refrigerada $\left(10^{\circ} \mathrm{C}\right)$. A formulação foi composta de concentrado proteico de soro, mix de vitaminas e minerais e goma guar como agente espessante. Os valores obtidos para a viscosidade mostraram diferença estatisticamente significativa $(P<0,05)$ entre os tempos de espessamento, sendo que as viscosidades no tempo de 10 minutos e após 24 horas variaram, respectivamente, $58 \pm 3 c P$ a $180 \pm 2$ cP na consistência de néctar; de $310 \pm 4$ Cp a $1084 \pm 3 c P$ na

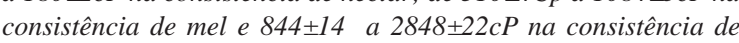
pudim. No entanto, a maioria das bebidas permaneceu dentro dos padrões internacionais sugeridos pela National Dysphagia Diet (NDD), para a consistência de alimentos destinados a pacientes disfágicos.

Palavras-chave: espessante alimentar, viscosidade, disfagia, gomas, shear rate.

\section{ABSTRACT}

Dysphagia is characterized by a dysfunction of the swallowing process, being a prevalent symptom of degenerative diseases such as multiple sclerosis and Parkinson's disease. Foods with modified texture and thickened beverages are traditionally used in the clinical treatment of dysphagia. This study aims to develop a formulation of food thickener with value nutritional aggregate, as well as evaluate its action on various liquid food, under different conditions of preparation time (10 and 120 minutes and 24 hours) temperature $\left(25^{\circ} \mathrm{C}\right)$ and refrigeration $\left(10^{\circ} \mathrm{C}\right)$. The formulation was composed of whey protein concentrate (WPC), mix of vitamins and minerals and guar gum as a thickening agent. The values obtained for the viscosity showed a difference statistically significant at the $5 \%$ level, between the times of thickening, ranging from $58 \pm 3 C P$ to $2848 \pm 3 C P$, as well as between different samples tested when compared to each other for the same time. However, viscosity measurements showed that the most beverages are in international standards suggested by the National Dysphagia Diet (NDD), for consistency of food for dysphagia patients.

Key words: food thickener, viscosity, dysphagia, shear rate.

\section{INTRODUÇÃO}

A deglutição é um processo complexo que envolve estruturas relacionadas com a cavidade oral, faringe, laringe e do esôfago, submetidos a um controle neuromuscular que permite o transporte do bolo alimentar da boca até o estômago, sem permitir a entrada de qualquer substância para a via aérea (ERTEKIN \& AYDOGDU, 2003). É definida como um processo altamente coordenado dividido em quatro fases, duas delas voluntárias, a oral preparatória e a oral; e duas involuntárias, a fase faríngea e a esofágica (SEO \& YOO, 2012).

Disfunções que venham a afetar a transferência do bolo alimentar da cavidade oral para faringe e interfiram no processo de deglutição resultam em disfagia, podendo ser causadas por uma deficiência neurogênica ou estrutural na cavidade oral ou do trato intestinal, o que resulta de uma ou mais

Instituto de Ciência e Tecnologia de Alimentos, Universidade Federal do Rio Grande do Sul (UFRGS), Av. Bento Gonçalves, 9500, Prédio 43.212, 91501-970, Porto Alegre, RS, Brasil. E-mail: simone.flores@ufrgs.br. *Autor para correspondência. 
patologias, tais como a doença de Parkinson, acidente vascular cerebral (AVC), entre outras (TAMURA, 2012).

De acordo com GERMAIN et al. (2006), os indivíduos disfágicos frequentemente sofrem de desnutrição, podendo vir acompanhada de outros problemas, como constipação, desidratação, úlceras e anorexia, geralmente causados pela baixa ingestão de nutrientes, considerados essenciais.

Promover nutrição adequada para indivíduos disfágicos é um desafio, no entanto, sua alimentação pode ser facilitada através da modificação na textura dos alimentos e espessamento dos líquidos ingeridos. Para evitar a desnutrição, nutricionistas e fonoaudiólogos devem ser envolvidos para avaliar a disfagia e recomendar as texturas mais adequadas para cada nível da doença (GERMAIN et al., 2006; SMITH, 2006).

Bebidas espessadas podem ser preparadas especialmente para o tratamento da disfagia e, em geral, os líquidos espessados são produzidos pela adição de um ou mais agentes espessantes, tais como amidos modificados ou gomas (GERMAIN et al., 2006; SOPADE et al., 2007). As gomas são aditivos alimentares com as funções de espessar e estabilizar, proporcionando textura desejada ao alimento com elevada viscosidade, mesmo em baixas concentrações (HONG et al., 2012).

O controle na viscosidade dos alimentos espessados é clinicamente importante para o manejo e tratamento de pacientes disfágicos, evitando os riscos de aspiração e retenção de alimentos na faringe após a deglutição (PAIK et al., 2004).

A falta de padrões claros para as dietas modificadas e para líquidos espessados levou à criação da National Dysphagia Diet Task Force (NDDTF), nos Estados Unidos, com a publicação em 2002 do guia da NationalDysphagia Diet (NDD) (GARCIA et al., 2005; MATTA, et al., 2006)

A NDD incluiu recomendações para rotulagem e taxas de viscosidade para cada um dos quatro níveis de consistência recomendados no tratamento da disfagia (fina, néctar, mel e pudim). A viscosidade dos líquidos espessados - mais especificamente a resistência a fluir - é expressa em centipoise (cP). As taxas de viscosidade dos alimentos líquidos, categorizadas pela NDD são: finos com viscosidade 1 a $50 \mathrm{cP}$, néctar de 51 a $350 \mathrm{cP}$, mel de 351 a $1750 \mathrm{cP}$ e pudim, superior a $1750 \mathrm{cP}$ (NDD, 2002). De acordo com GERMAIN et al. (2006) as viscosidades sugeridas pela NDD não foram clinicamente estabelecidas como parâmetros fixos, no entanto, podem ser usados como uma unidade de referência, devido à falta de padrões claros.
Uma vez que a consistência da maioria dos líquidos consumidos sofre variações em função da taxa de fluxo, as medidas de viscosidade são geralmente expressas a uma taxa de cisalhamento específica (shear rate) (STEELE et al., 2003). Com base em investigações realizadas, o NDDTF selecionou taxa de cisalhamento de $50 \mathrm{~s}^{-1}$ e temperatura de $25 \pm 1^{\circ} \mathrm{C}$ como padrões para a medida da viscosidade do NDD (2002).

Levando em consideração os transtornos causados pela disfagia e os sérios problemas de desnutrição que acometem tais indivíduos, dois objetivos foram propostos para este trabalho: (1) Formular um espessante alimentar, utilizando goma como agente espessante, adicionado de proteínas, vitaminas e minerais; (2) Avaliar a viscosidade do produto formulado, adicionado em diferentes alimentos líquidos, testando a influência do tempo e temperatura, sobre a estabilidade das viscosidades obtidas, de acordo com os níveis de consistência recomendados.

\section{MATERIAL E MÉTODOS}

\section{Matéria-prima}

Para formulação do produto, utilizaram-se como matéria-prima $68 \%$ de concentrado proteico de soro ( $80 \%$ de proteína), obtidos de acordo com Pagnoet al. (2009); 2\% do mix de vitamina (vitaminas $\mathrm{E}, \mathrm{A}, \mathrm{D}, \mathrm{K}, \mathrm{B} 12$, ácido pantoténico (B5), piridoxina (B6), tiamina (B1), riboflavina (B2), ácido fólico (B9) e biotina e minerais ( $\mathrm{Ca}, \mathrm{Fe}, \mathrm{P}, \mathrm{Zn}, \mathrm{K}, \mathrm{Cl}, \mathrm{Mg}, \mathrm{Mn}, \mathrm{Cu}$, I, Cr, Se, F), fornecido pela empresa Fortitech Sul ${ }^{\circledR}$ e $30 \%$ goma de guar, utilizado como agente espessante, fornecido pela empresa HexusFoodIngredients.

A fim de determinar a concentração de agente espessante necessário para a adição à formulação, a viscosidade aparente em centipoise (cP) foi medida em viscosímetro Brookfield, modelo DVII+PRO, com diferentes concentrações de goma de guar, variando de $0,4 \%$ a até $2,0 \%$, dissolvido em água destilada. As medidas foram realizadas à temperatura de $25^{\circ} \mathrm{C}$ com taxa de cisalhamento a $50 \mathrm{~s}^{-1}$ (GARCIA et al., 2005 ).

Testes de aplicação

Os líquidos utilizados como amostras para os testes com o produto formulado foram: leite integral (Santa Clara ${ }^{\circledR}$ ), sucos de maçã $\left(\right.$ Tial $\left.^{\circledR}\right)$, uva $\left(\right.$ Jandaia $\left.^{\circledR}\right)$ e laranja $\left(\right.$ Maguary $\left.{ }^{\circledR}\right)$. Esses alimentos são comumente espessados e servidos a indivíduos disfágicos. Para o preparo das amostras, $600 \mathrm{~mL}$ dos líquidos citados acima foram adicionados em Becker 
de vidro e o produto formulado foi adicionado lentamente sob agitação até completa dissolução.

As medidas de viscosidade foram realizadas em viscosímetro Brookfield, modelo DV-II+PRO, utilizando spindle LV4 e expressas em Centipoise (cP). O equipamento estava conectado ao computador para controle e coleta dos dados, através do software Rheocalc V3. De acordo com as recomendações da National Dysphagia Diet (NDD), as medidas foram realizadas com taxa de cisalhamento (shear rate) de 50. $\mathrm{s}^{-1}$ (NDD, 2002).

Com a finalidade de avaliar o comportamento do espessante formulado frente a diferentes condições, medidas da viscosidade foram realizadas em diferentes condições de tempo e temperatura. Primeiramente, as medidas foram realizadas 10 minutos, considerando um consumo imediato após preparo dos alimentos e duas horas após seu preparo, levando em consideração uma provável demora para que os alimentos cheguem até os pacientes, todos a uma temperatura de $25 \pm 2^{\circ} \mathrm{C}$. As amostras, já espessadas, foram então armazenadas sob refrigeração por $24 \mathrm{~h}$ e novas medidas foram realizadas em temperatura de $10 \pm 2^{\circ} \mathrm{C}$, a fim de avaliar o comportamento do produto formulado frente a um possível armazenamento do alimento espessado, sendo assim fornecido aos pacientes apenas no dia seguinte. Tais parâmetros foram baseados em outros trabalhos da literatura que realizaram a avaliação de alimentos espessados para indivíduos disfágicos frente as mesmas condições (GARCIA et al., 2005; GERMAIN et al., 2006).

\section{Análise Estatística}

Os resultados foram expressos na forma de média e desvio padrão para cada amostra. Os dados foram analisados pela ANOVA e as médias comparadas pelo teste de Tukey ao nível de 5\% de significância (BENDER et al., 1982).

\section{RESULTADOS E DISCUSSÃO}

As medidas da viscosidade aparente dos testes preliminares realizados com o agente, variaram entre $96,71 \mathrm{cP}$ para a concentração de $0,4 \%$, até $2142,20 \mathrm{cP}$ à concentração de $1,2 \%$. SOPADE et al. (2007) avaliaram a viscosidade de espessantes comerciais a base de goma e amido de milho modificado, dispersos em água e sucos, e observaram aumento da viscosidade com aumento da concentração do espessante.

A porção de cada composto adicionado no produto teve por objetivo realizar suplementação nutricional de proteínas, vitaminas e sais minerais, seguindo as recomendações de Ingestão Diária de Referência Brasileira (BRASIL, 2003). A formulação final foi composta de $68 \%$ de concentrado de proteína de soro, $2 \%$ de mix de vitaminas e sais minerais e $30 \%$ de goma guar.

O espessante utilizado na formulação do produto em questão foi goma guar, uma fibra solúvel, que tem a capacidade de formar soluções altamente viscosas em baixas concentrações. Dessa forma, a quantidade adicionada na formulação foi baixa e o consumo do espessante formulado não aumenta significativamente a ingestão de fibras, não ocasionando portanto a complexação de outros nutrientes. No entanto, sabe-se que tais fibras agem na diminuição do colesterol sanguíneo e também são eficazes na diminuição da hiperglicemia.

Em estudo realizado por HANNAN et al. (2007), foi verificado que a administração de fibra dietética solúvel em ratos com diabetes tipo I e II melhorou a tolerância à glicose. Concluindo que o efeito "anti-diabético" das fibras solúveis se deve à inibição da digestão e absorção da glicose e ao aumento da ação periférica da insulina. Portanto, o indivíduo diabético se beneficia da menor absorção da glicose resultante da ingestão aumentada de fibras.

Por meio de testes preliminares, calculouse a quantidade em $(\mathrm{g})$ ou porção, necessária do produto formulado para ser adicionado a um copo $(250 \mathrm{~mL})$ dos alimentos líquidos a fim de atingir os níveis de consistência sugeridos pela NDD (2002) (néctar, mel e/ou pudim), sendo, respectivamente, 4,2; 6,7 e 9,2g para a consistência de néctar, mel e pudim. $\mathrm{O}$ valor energético atribuído a cada porção deve-se principalmente ao teor de proteína, uma vez que os demais constituintes apresentam baixa quantidade, obtendo assim suplementação de 9; 15 e 20kcal, respectivamente, para cada porção, melhorando a ingestão calórica, além da suplementação de vitaminas e minerais que podem ser visualizadas na tabela 1 .

Em estudo realizado por GERMAIN et al. (2006), avaliaram-se o ganho de peso e aumento de massa corpórea de pacientes disfágicos que receberam, além da alimentação tradicional oferecida, bebida espessada. Os autores observaram aumento de peso no grupo que recebeu bebida espessada, em comparação com o grupo controle, consequentemente, aumento do índice de massa corpórea.

Na tabela 2, podem-se observar as médias de viscosidade expressas em centipoise (cP), dos alimentos líquidos espessados (leite, sucos de maçã, suco de uva e suco de laranja) em cada nível de 
Tabela 1- Composição de vitamina e minerais de acordo com aIngestão Diaria Recomendada para todos os níveis de consistência (néctar, mel e pudim)

\begin{tabular}{|c|c|c|c|c|c|c|}
\hline & \multirow{2}{*}{------------- } & 4,2g----. & \multirow{2}{*}{\multicolumn{2}{|c|}{$\begin{array}{r}\text { \% } \\
\text { \% IDR }\end{array}$}} & \multirow{2}{*}{\multicolumn{2}{|c|}{$\begin{array}{r}\text {--ordim 9,2g----- } \\
\% \text { IDR }\end{array}$}} \\
\hline & & $\%$ IDR & & & & \\
\hline Cálcio & $15 \mathrm{mg}$ & $1,50 \%$ & $23,90 \mathrm{mg}$ & $2,40 \%$ & $32,8 \mathrm{mg}$ & $3,50 \%$ \\
\hline Fósforo & $8,2 \mathrm{mg}$ & $1,20 \%$ & $13,15 \mathrm{mg}$ & $1,90 \%$ & $18 \mathrm{mg}$ & $2,60 \%$ \\
\hline Ferro & $0,30 \mathrm{mg}$ & $1,80 \%$ & $0,41 \mathrm{mg}$ & $2,90 \%$ & $0,56 \mathrm{mg}$ & $4,00 \%$ \\
\hline Zinco & $69,30 \mu \mathrm{g}$ & $1,00 \%$ & $111 \mu \mathrm{g}$ & $1,60 \%$ & $152 \mu \mathrm{g}$ & $2,20 \%$ \\
\hline Manganês & $26,46 \mu \mathrm{g}$ & $1,20 \%$ & $42,21 \mu \mathrm{g}$ & $1,80 \%$ & $58 \mu \mathrm{g}$ & $2,50 \%$ \\
\hline Cobre & $12,60 \mu \mathrm{g}$ & $1,40 \%$ & $20,10 \mu \mathrm{g}$ & $2,20 \%$ & $28 \mu \mathrm{g}$ & $3,10 \%$ \\
\hline Selênio & $0,43 \mu \mathrm{g}$ & $0,80 \%$ & $0,70 \mu \mathrm{g}$ & $1,20 \%$ & $0,94 \mu \mathrm{g}$ & $1,70 \%$ \\
\hline Molibdênio & $0,33 \mu \mathrm{g}$ & $0,70 \%$ & $0,55 \mu \mathrm{g}$ & $1,20 \%$ & $0,73 \mu \mathrm{g}$ & $1,60 \%$ \\
\hline Niacina $\left(B_{3}\right)$ & $0,13 \mathrm{mg}$ & $0,80 \%$ & $0,20 \mathrm{mg}$ & $1,30 \%$ & $0,28 \mathrm{mg}$ & $1,70 \%$ \\
\hline Ac. Pantotênico & $67,20 \mu \mathrm{g}$ & $1,30 \%$ & $107,20 \mu \mathrm{g}$ & $2,10 \%$ & $147,20 \mu \mathrm{g}$ & $2,90 \%$ \\
\hline Piridoxina $\left(\mathrm{B}_{6}\right)$ & $29,40 \mu \mathrm{g}$ & $2,30 \%$ & $46,90 \mu \mathrm{g}$ & $3,60 \%$ & $64,40 \mu \mathrm{g}$ & $5,00 \%$ \\
\hline $\operatorname{Tiamina}\left(\mathrm{B}_{1}\right)$ & $25,20 \mu \mathrm{g}$ & $2,10 \%$ & $40,20 \mu \mathrm{g}$ & $3,40 \%$ & $55,20 \mu \mathrm{g}$ & $4,60 \%$ \\
\hline Riboflavina $\left(\mathrm{B}_{2}\right)$ & $25,20 \mu \mathrm{g}$ & $1,90 \%$ & $40,20 \mu \mathrm{g}$ & $3,10 \%$ & $55,20 \mu \mathrm{g}$ & $4,20 \%$ \\
\hline Ácido Fólico & $8,40 \mu \mathrm{g}$ & $2,10 \%$ & $13,40 \mu \mathrm{g}$ & $3,40 \%$ & $18,40 \mu \mathrm{g}$ & $4,60 \%$ \\
\hline Biotina & $0,84 \mu \mathrm{g}$ & $2,80 \%$ & $1,34 \mu \mathrm{g}$ & $4,50 \%$ & $1,84 \mu \mathrm{g}$ & $6,10 \%$ \\
\hline Vitamina $\mathrm{B}_{12}$ & $0,11 \mu \mathrm{g}$ & $4,40 \%$ & $0,17 \mu \mathrm{g}$ & $7,00 \%$ & $0,23 \mu \mathrm{g}$ & $9,60 \%$ \\
\hline Vitamina $\mathrm{E}$ & $0,63 \mathrm{mg}$ & $4,20 \%$ & $1,01 \mathrm{mg}$ & $6,70 \%$ & $1,40 \mathrm{mg}$ & $9,20 \%$ \\
\hline Vitamina A & $33,60 \mu \mathrm{g}$ & $3,70 \%$ & $53,6 \mu \mathrm{g}$ & $6,00 \%$ & $73,60 \mu \mathrm{g}$ & $8,20 \%$ \\
\hline Vitamina D & $0,03 \mu \mathrm{g}$ & $0,50 \%$ & $0,04 \mu \mathrm{g}$ & $0,80 \%$ & $0,06 \mu \mathrm{g}$ & $1,20 \%$ \\
\hline Vitamina $\mathrm{K}$ & $3,1 \mu \mathrm{g}$ & $4,80 \%$ & $5,03 \mu \mathrm{g}$ & $7,70 \%$ & $6,9 \mu \mathrm{g}$ & $10,60 \%$ \\
\hline
\end{tabular}

consistência (néctar, mel e pudim) e para cada tempo de espessamento (10 minutos, 2 e 24 horas). Valores menores representam líquidos menos viscosos ou mais fluídos. Pode-se observar que as médias de viscosidade aparente das amostras, foram dependente do tipo de amostra utilizada e do tempo de ação do agente espessante.

As médias da viscosidade das amostras foram comparadas aos limites sugeridos pela National Dysphagia Diet (NDD) (GARCIA et al., 2005). Para o nível de consistência de néctar $(51-350 \mathrm{cP})$, todas as amostras, independente do tempo de espessamento, permaneceram dentro dos limites, variando da menor viscosidade, de $58 \mathrm{cP}$, para o suco de uva no tempo de 10 minutos, até $180 \mathrm{cP}$, para o suco de maçã após 24 horas, como pode ser observado na tabela 2.

Para a consistência tipo mel (351-1750cP), apenas o suco de uva com viscosidade média de $310 \mathrm{cP}$ no tempo de 10 minutos permaneceu abaixo do índice sugerido, as demais amostras encontraram-se dentro da faixa estipulada, com viscosidade máxima de 1084 cP para o leite após 24 horas.

$\mathrm{Na}$ consistência de pudim $(>1750 \mathrm{cP})$, todas as amostras ficaram abaixo do índice sugerido no tempo de 10 minutos, variando de $1199 \mathrm{cP}$ para a viscosidade do leite a $844 \mathrm{cP}$ para o suco de uva. Já, após duas horas, com exceção da amostra de leite que continuou abaixo do limite mínimo sugerido, todas as demais amostras permaneceram dentro do limite sugerido pela NDD para a viscosidade avaliada.

GERMAIN et al. (2006), ao avaliarem a viscosidade de bebidas espessadas, tradicionalmente usadas para disfagia, nas condições em que eram oferecidas aos pacientes $\left(8^{\circ} \mathrm{C}\right)$, observaram que grande parte das amostras testadas não estava de acordo com níveis de consistência sugeridos pela National Dysphagia Diet (NDD, 2002). De 30 amostras analisadas, apenas 9 encontravam-se dentro dos limites.

Ao comparar as médias de viscosidade nos diferentes tempos de espessamento para cada nível de consistência, pode-se observar aumento da viscosidade com diferença estatisticamente significativa em todos os casos, como pode ser visualizado na tabela 2.

Segundo GARCIA et al. (2005), este resultado pode ser explicado pelo fato de o espessante continuar absorvendo água e hidratandose durante um certo período de tempo, aumentando com isso a viscosidade das amostras. Os mesmos autores, avaliando a ação de espessantes alimentares comerciais em alimentos, como sucos, café e leite, ao medirem a viscosidade após 10 e 30 minutos (tempo padrão sugerido pelos fabricantes), observaram 
Tabela 2 - Médias e desvio padrão da viscosidade das amostras espessadas, com consistência de Néctar, Mel e Pudim, expressas em centipoise (cP), e tempo de espessamento, com taxa de cisalhamento de $50 \mathrm{~s}^{-1}$

\begin{tabular}{|c|c|c|c|c|}
\hline Tempo de espessamento para cada consistência & Leite & Suco de maçã & Suco de uva & Suco de laranja \\
\hline \multicolumn{5}{|l|}{ Néctar } \\
\hline $10 \mathrm{~min}$ & $89 \pm 1^{\mathrm{cA}}$ & $68 \pm 2^{\mathrm{cB}}$ & $58 \pm 3^{\mathrm{cC}}$ & $60 \pm 2^{\mathrm{cC}}$ \\
\hline $2 \mathrm{~h}$ & $153 \pm 2^{\mathrm{bA}}$ & $163 \pm 4^{\mathrm{bA}}$ & $136 \pm 4^{\mathrm{bB}}$ & $157 \pm 4^{\mathrm{bA}}$ \\
\hline $24 \mathrm{~h}$ & $166 \pm 3^{\text {а В }}$ & $180 \pm 2^{\mathrm{aA}}$ & $151 \pm 0.4^{\mathrm{aC}}$ & $168 \pm 2^{\text {а в }}$ \\
\hline \multicolumn{5}{|l|}{ Mel } \\
\hline $10 \mathrm{~min}$ & $515 \pm 35^{\mathrm{cA}}$ & $412 \pm 3^{\mathrm{cB}}$ & $310 \pm 4^{\mathrm{aC}}$ & $424 \pm 7^{\mathrm{cB}}$ \\
\hline $2 \mathrm{~h}$ & $827 \pm 7^{\mathrm{bB}}$ & $867 \pm 10^{\mathrm{bA}}$ & $834 \pm 1^{\mathrm{bB}}$ & $837 \pm 5^{\text {b в }}$ \\
\hline $24 \mathrm{~h}$ & $1084 \pm 3^{\text {a B }}$ & $1042 \pm 7^{\text {a A }}$ & $1001 \pm 3^{\mathrm{cC}}$ & $998 \pm 3^{\text {a C }}$ \\
\hline \multicolumn{5}{|l|}{ Pudim } \\
\hline $10 \mathrm{~min}$ & $1199 \pm 59^{\mathrm{cA}}$ & $904 \pm 19^{\mathrm{cBCD}}$ & $844 \pm 14^{\mathrm{cC}}$ & $949 \pm 16^{\mathrm{cD}}$ \\
\hline $2 \mathrm{~h}$ & $1658 \pm 14^{\mathrm{bB}}$ & $1841 \pm 27^{\mathrm{bA}}$ & $1797 \pm 29^{\mathrm{bA}}$ & $1801 \pm 22^{\mathrm{bA}}$ \\
\hline $24 \mathrm{~h}$ & $2848 \pm 22^{\text {a A }}$ & $2278 \pm 21^{\text {a B }}$ & $2328 \pm 24^{\text {a B }}$ & $2198 \pm 22^{\text {a C }}$ \\
\hline
\end{tabular}

Letras minúsculas diferentes na mesma coluna indicam diferença estatisticamente significativa $(\mathrm{P} \leq 0,05)$ pelo teste de Tukey entre os tempos de espessamento. Letras maiúsculas diferentes na mesma linha indicam diferença estatisticamente significativa $(\mathrm{P} \leq 0,05)$ pelo teste de Tukey entre as amostras.

aumento da viscosidade na maioria das amostras, nos níveis de consistência analisados.

Vale ressaltar que, no presente estudo, nos tempos 10 minutos e duas horas, as medidas da viscosidade foram realizadas à temperatura ambiente. Após 24 horas, com as amostras estocadas em temperatura de refrigeração, as medidas foram realizadas na temperatura de $10 \pm 1^{\circ} \mathrm{C}$. ADELEYE \& RACHAL (2007), ao examinarem a influência da temperatura frente às propriedades reológicas, observaram que as mesmas amostras possuíam diferença estatisticamente significativa na viscosidade, ao serem avaliadas a 10 e $20^{\circ} \mathrm{C}$, obtendo maior viscosidade em menores temperaturas, independente do nível de consistência. De acordo com HONG et al. (2012), a temperatura influencia na viscosidade das amostras, obtendo, de maneira geral, viscosidade mais elevada em temperaturas mais baixas.

Ao comparar as amostras entre si, dentro do mesmo tempo e menos nível de consistência, observam-se diferentes valores na viscosidade (Tabela 2). Essa diferença pode ter ocorrido devido a diferentes constituintes contidos em cada amostra que causam forte impacto sobre os resultados da viscosidade final. De acordo com SOPADE et al. (2008), podem ocorrer interações entre o agente espessante (goma guar) e alguns dos constituintes da amostra, bem como aumento no teor de sólidos, o que causa maior interação intermolecular entre os sólidos, proporcionando aumento na viscosidade. $\mathrm{O}$ baixo $\mathrm{pH}$ dos alimentos, devido à presença de ácidos, como nos sucos, é conhecido por afetar a reologia de espessantes a base de amidos e gomas, podendo ter um efeito negativo, reduzindo a viscosidade (SOPADE, et al., 2007). Além disso, GARCIA et al. (2005) cita que o valor de $\mathrm{pH}$ dos alimentos pode interagir na viscosidade dos alimentos, a acidez pode provocar degradação das ligações químicas das gomas, reduzindo sua viscosidade.

Dentre as amostras analisadas, o leite e o suco de maçã apresentaram as maiores médias de viscosidade, independente do nível de consistência ou tempo de espessamento. De acordo com GARCIA et al. (2005), o leite contém minerais e outros ingredientes que podem interagir com o espessante, resultando em maior capacidade de espessamento, já, para o sucos, o alto teor de sólidos pode resultar em maiores viscosidades. Segundo os mesmos autores, o valor de $\mathrm{pH}$ dos alimentos pode ter alguma interação na viscosidade dos alimentos, através da degradação as ligações química das gomas, provocando redução na viscosidade.

\section{CONCLUSÃO}

O produto formulado neste trabalho, além de atuar como espessante para líquidos, destinados a indivíduos disfágicos, também contribuiu para suplementação nutricional de proteínas, vitaminas e minerais. Os testes realizados com o produto formulado demonstraram sua eficiência para espessar diferentes alimentos líquidos, comumente utilizados no manejo de pacientes com déficit de deglutição, tornado-as com consistência adequada para sua 
alimentação. Observou-se que praticamente todos os alimentos testados permaneceram dentro dos limites de viscosidade sugeridos pela National Dysphagia Diet (NDD). Com exceção dos alimentos na consistência de pudim, todos ficaram com a consistência abaixo do limite mínimo indicado para o tempo de 10 minutos, sugerindo-se um aumento na porção para a atingir a consistência de pudim nos primeiros minutos de preparo. Dessa maneira, pode-se recomendar que o tempo mais adequado fosse o de duas horas após o preparo dos alimentos, proporcionado assim maior garantia de que os alimentos atinjam os níveis de consistência sugeridos. No entanto, mais trabalhos devem ser realizados a fim de aperfeiçoar a formulação e determinar um tempo padrão de espessamento dos alimentos. Recomendase futuros estudos com aplicação de análise sensorial de amostras espessadas e testes com indivíduos disfágicos.

\section{REFERENCIAS}

ADELEYE, B., RACHAL, C. Comparison of the rheological properties of ready-to-serve and powdered instant food-thickened beverages at different temperatures for dysphagic patients. Journal of the American Dietetic Association, Chicago, v.107, n.7, p.1176-82, 2007. Disponível em: <http://www.sciencedirect. com/science/article/pii/S0002822307005925>. Acesso em: 08 ago. 2012. doi: 10.1016/j.jada.2007.04.011.

BENDER, F.E. et al. Statistical methods for food and agriculture. Westport:Avi, 1982. 345p.

BRASIL. Resolução RDC n.360, de 23 de dezembro de 2003. Regulamento Técnico Sobre Rotulagem Nutricional de Alimentos Embalados. 2003. Agência Nacional de Vigilância Sanitária. Disponível em: <http://www.anvisa.gov.br/legis/ resol/2003/rdc/360_03rdc.htm>. Acesso em: 22 ago. 2012.

ERTEKIN, C.; AYDOGDU, I.Neurophysiology of swallowing. Clinical Neurophysiology, Amsterdam, v.114, n.12, p.2226-2244, 2003. Disponível em: <http://www.sciencedirect.com/science/ article/pii/S1388245703002372>. Acessoem: 06 ago. 2012. doi: 10.1016/S1388-2457(03)00237-2

GARCIA, J.M. et al. Viscosity measurements of nectar- and honeythick liquids: product, liquid, and time comparisons. Dysphagia, New York, v.20, n.4, p.325-335, 2005. Disponível em: <http://link. springer.com/article/10.1007/s00455-005-0034-9/fulltext.html $>$. Acesso em: 05 ago. 2012. doi: 10.1007/s00455-005-0034-9.

GERMAIN, I. et al. Novel dysphagia diet improves the nutrient intake of institutionalized elders. Journal of the American Dietetic Association, Chicago, v.106, n.10, p.1614-1623, 2006. Disponível em: <www.sciencedirect.com/science/article/pii/ S0002822306016968>. Acesso em: 09 ago. 2012. doi: 10.1016/j. jada.2006.07.008.

GERMAIN, I. et al. Rheological characterization of thickened beverages used in the treatment of dysphagia. Journal of Food Engineering, Essex, v.73, n.1, p.64-74, 2006. Disponível em: <http://
www.sciencedirect.com/science/article/pii/S0260877405000403>. Acesso em: 09 ago. 2012. doi: 10.1016/j.jfoodeng.2005.01.006.

HONG, S.R. et al. Flow behaviors of commercial food thickeners used for the management of dysphagia: effect of temperature. International Journal of Food Engineering, Inglaterra. v.8, n.2, artigo 8, p.1-8, 2012. Disponível em: <www.degruyter.com/ view/j/ijfe.2012.8.issue-2/1556-3758.2215/1556-3758.2215.xml>. Acesso em: 10 Ago. 2012. doi: 10.1515/1556-3758.2215.

HANNAN, J.M. et al. Aqueous extracts of husks of Plantagoovata reduce hyperglycaemia in type 1 and type 2 diabetes by inhibition of intestinal glucose absorption. British Journal of Nutrition, Cambridge, v.96, n.1, p.131-137, 2006. Disponível em: <www. ncbi.nlm.nih.gov/pubmed/16870001>. Acesso em: 11 ago. 2012. doi: 10.1079/BJN20061819.

JOTZ, G.P.; DORNELLES, S.Fisiologia da deglutição.In: JOTZ, G.P.; DORNELLES, S. Otorrinolaringologia: princípios e práticas. 2.ed. São Paulo: Artmed, 2006.p.753-756.

MATTA, Z.M.S. et al. Sensory characteristics of beverages prepared with commercial thickeners used for dysphagia diets. Journal of the American Dietetic Association, Chicago, v.106, n.7, p.1049-54, 2006. Disponível em: <www.ncbi.nlm.nih.gov/ pubmed/16815121>. doi: 10.1016/j.jada.2006.04.02.

NDD. National Dysphagia Task Force: national dysphagia diet: Standardization for optimal care. Chicago, IL: American Dietetic Association, 2002. 47 p.

PAIK, N.J. et al. Obtenção de concentrados protéicos de soro de leite e caracterização de suas propriedades funcionais tecnológicas. Alimentos e Nutrição, Araraquara, v.20, n.2, p.231-239, 2009. Disponível em: <http://serv-bib.fcfar.unesp.br/seer/index.php/ alimentos/article/view/1052/803 >. Acesso em: 20 fev. 2013.

LEE, E.K. et al. Categorization of dysphagia diets with the line spread test. Archives of Physical Medicine and Rehabilitation, Philadelphia, v.85, n.5, p.857-861, 2004. Disponível em: < www. sciencedirect.com/science/article/pii/S0003999303010220>. Acesso em: 23 ago. 2012. doi: 10.1016/j.apmr.2003.08.079.

SEO, C.W.; YOO, B. Steady and dynamic shear rheological properties of gum-based food thickeners used for diet modification of patients with dysphagia: effect of concentration. Dysphagia, v.28, n.2, p. 205-211, 2012. Disponível em: <http://link.springer.com/ article/10.1007\%2Fs00455-012-9433-x?LI=true >. Acesso em: $27 \mathrm{fev}$. 2013. doi: 10.1007/s00455-012-9433-x.

SMITH, P. Nutrition, hydration, and dysphagia in long-term care: differing opinions on the effects of aspiration. Journal of the American Medical Directors Association, St. Louis, v.7, n.9, p.545-549, 2006. Disponível em: <www.ncbi.nlm.nih.gov/pubmed/17095418>. Acesso em: 03 de mar. de 2012. doi: 10.1016/j.jamda.2006.03.008.

SOPADE, P.A. et al. Rheological characterization of food thickeners marketed in Australia in various media for the management of dysphagia. III. Fruit juice as a dispersing medium. Journal of Food Engineering, Essex, v.86, n.4, p.604-615, 2008. Disponível em: <http://193.146.160.29/gtb/sod/usu/\$UBUG/ repositorio/10301094_Sopade2.pdf $>$. Acesso em: 20 set. 2012. doi: 10.1016/j.jfoodeng.2007.11.013.

SOPADE, P.A. et al. Rheological characterization of food thickeners marketed in Australia in various media for the management of 
dysphagia. I: water and cordial.Journal of Food Engineering, Essex, v.79, n.1, p.69-82, 2007. Disponível em: <www. sciencedirect.com/science/article/pii/S0260877406001026>. Acesso em: 06 ago. 2012. doi: 10.1016/j.jfoodeng.2006.01.045.

STEELE, C.M. et al. The rheology of liquids: a comparison of clinicians' subjective impressions and objective measurement. Dysphagia, New York, v.18, n.3, p.182-195, 2003. Disponível em:
$<$ http://link.springer.com/article/10.1007\%2Fs00455-002-0104-1>. Acesso em: 20 set. 2012.doi: 10.1007/s00455-002-0104-1.

TAMURA, F.et al. Tongue thickness relates to nutritional status in the Elderly. Dysphagia, New York v.27, n.4, p.556-561, 2012. Disponível em: <http://link.springer.com/ article/10.1007\%2Fs00455-012-9407-z?LI=true>. Acesso em: 26 fev. 2013.doi: 10.1007/s00455-012-9407-z.

Ciência Rural, v.44, n.4, abr, 2014. 